\title{
A Cellular Automata-based Model for Simulating Restitution Property in a Single Heart Cell
}

\author{
Seyed Hojjat Sabzpoushan, Fateme Pourhasanzade \\ Department of Biomedical Engineering, The Research Laboratory of Biomedical Signals and Sensors, Iran University of Sciences and Technology \\ (I.U.S.T), Tehran, Iran
}

A B S T R A C T

\begin{abstract}
Ventricular fibrillation is the cause of the most sudden mortalities. Restitution is one of the specific properties of ventricular cell. The recent findings have clearly proved the correlation between the slope of restitution curve with ventricular fibrillation. This; therefore, mandates the modeling of cellular restitution to gain high importance. A cellular automaton is a powerful tool for simulating complex phenomena in a simple language. A cellular automaton is a lattice of cells where the behavior of each cell is determined by the behavior of its neighboring cells as well as the automata rule. In this paper, a simple model is depicted for the simulation of the property of restitution in a single cardiac cell using cellular automata. At first, two state variables; action potential and recovery are introduced in the automata model. In second, automata rule is determined and then recovery variable is defined in such a way so that the restitution is developed. In order to evaluate the proposed model, the generated restitution curve in our study is compared with the restitution curves from the experimental findings of valid sources. Our findings indicate that the presented model is not only capable of simulating restitution in cardiac cell, but also possesses the capability of regulating the restitution curve.
\end{abstract}

Key words: Cellular automata, restitution property, sudden cardiac death, ventricular fibrillation

\section{INTRODUCTION}

Sudden cardiac death is a major cause of mortality in the industrialized world, ${ }^{[1]}$ and ventricular fibrillation (VF) is the leading cause of sudden cardiac death. ${ }^{[2]}$ An electrical wavebreak in the heart tissue may lead to VF. According to the so-called restitution hypothesis, rate-dependent alteration of action potential duration (APD) and refractoriness (R) are believed to be important determinants of the wavebreak. ${ }^{[3]}$ The changes in APD that accompany the changes in rate, reflect the dependence of APD on the preceding diastolic interval (DI), a relationship characterized by the electrical restitution curve. ${ }^{[3]}$ All of these mean that the modeling and simulating of restitution property in the cardiac cell is very important.

Cellular Automata (CA) may be considered as a method for modeling discrete dynamic systems. ${ }^{[4]}$ A CA consists of a discrete system of lattice sites (cells) having various initial values. These cells evolve in discrete time steps, as each cell assumes a new state based on the rules, that is, the states of its local neighborhood and a finite number of previous time steps. ${ }^{[5]}$ The neighborhood is described by specifying the set of cells that are the neighbors of a given cell. Figure 1 depicts two kinds of neigborhoods.

A CA lattice may be 1-D or multidimensional. For 2-D $\mathrm{CA}$, there are several possible lattices and neighborhood structures. For a squarelattice, two types of neighborhoods are typically used; the generalized 'Von Neumann' neighborhood and the 'Moore' neighborhood. ${ }^{[6]}$

Action potential duration restitution is an important electrical property of the cardiac cells. If a cell which is still in the refractory state is re-stimulated by an adequate large stimulus ( $\mathrm{S} 2$ in Figure 2), the generated APD; $\mathrm{APD}_{\mathrm{n}+1}$ will be shorter than that in the normal case, $\mathrm{APD}_{\mathrm{n}}$, that is, when the cell is stimulated after complete refractory.

The action potential duration restitution property refers to the relationship between $A P D_{n+1}$ and the previous diastolic interval $\mathrm{DI}_{\mathrm{n}}$, as shown in Figure 2. DI is defined by the duration between the end of the cell's re-polarization and the next applied stimulation [Figure 2].

The plot of $\mathrm{APD}_{\mathrm{n}+1}$ versus $\mathrm{DI}_{\mathrm{n}}$ is called the restitution curve [Figure 3]. ${ }^{\mid 9]}$ 
Restitution curve may be explained as a function $f$ :

$A P D_{n+1}=f\left(D I_{n}\right)$

Experimental measurements and observations confirmed that the restitution curve may be fitted by a sigmoid function like: ${ }^{[3]}$

$$
A P D_{n+1}=a+\frac{b}{1+e^{\frac{-(D I-c)}{d}}}
$$

There are different methods for experimental plotting of the restitution curve, like the S1-S2 standard and the dynamical restitution method. ${ }^{[3]}$

Action potential duration is usually explained as $\mathrm{APD}_{95}$ or $A P D_{90} \cdot A_{P D}$ is defined as the time distance between the S2 stimulus (beginning of AP) and 95\% of the cell's full repolarization. In this research, $\mathrm{APD}_{95}$ has been used. Regarding Figure 2, DI is defined as follows:

$\mathrm{DI}=(\mathrm{S} 1-\mathrm{S} 2$ interval $)-\left(\mathrm{APD}_{95}\right.$ caused by last $\left.\mathrm{S} 1\right)$

\section{AIMS}

In the literature, mainly square lattices, with nearest neighbor interactions are studied. However, triangle and hexagonal lattices are also possible. ${ }^{[4]}$ Figure 1 illustrates two types of neighborhoods that are generally used in literature..$^{77}$

In this article a model based on CA is proposed. This model can be used for simulating the restitution phenomena in a ventricular cell.

This article is organized as follows: Section 2 reviews the restitution property. In Section 3 the proposed automata model is introduced, as also the method of implementation of the restitution property in the proposed model. Section 4 includes the simulation results and model validation investigation. Section 5 has the conclusions and remarks.

\section{MATERIALS AND METHODS}

Presume a square lattice with $\mathrm{M} \times \mathrm{M}$ cells. Two state variables are assigned to each cell; one for the action potential variable $\left(\mathrm{AP}_{\mathrm{mn}}^{\mathrm{t}}\right)$ and the other for the refractory variable $\left(\mathrm{R}_{\mathrm{mn}}^{\mathrm{t}}\right)$, where $\mathrm{m}, \mathrm{n}$, and $\mathrm{t}$ denote the column number, row number, and the time step, respectively. The amplitude of each state variable takes a value from 0 to $\mathrm{N}-1$. The variable $\mathrm{S}_{\mathrm{mn}}^{\mathrm{t}}$ stands for the stimulus applied to the cell, which is in the coordinate $(\mathrm{m}, \mathrm{n})$ in time t. Table 1 summarizes the description of the other parameters.

The transition rule (automata rule) in the proposed model is defined as follows:

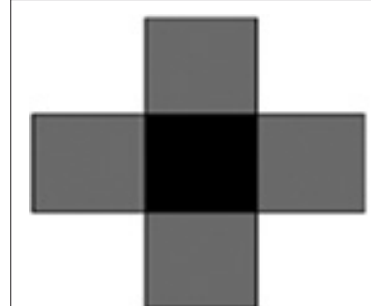

(a)

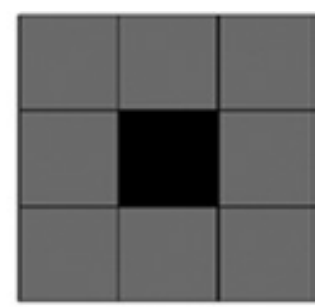

(b)
Figure I: Two kinds of neighborhoods. (a) Von Neumann; (b) Moore ${ }^{[7]}$

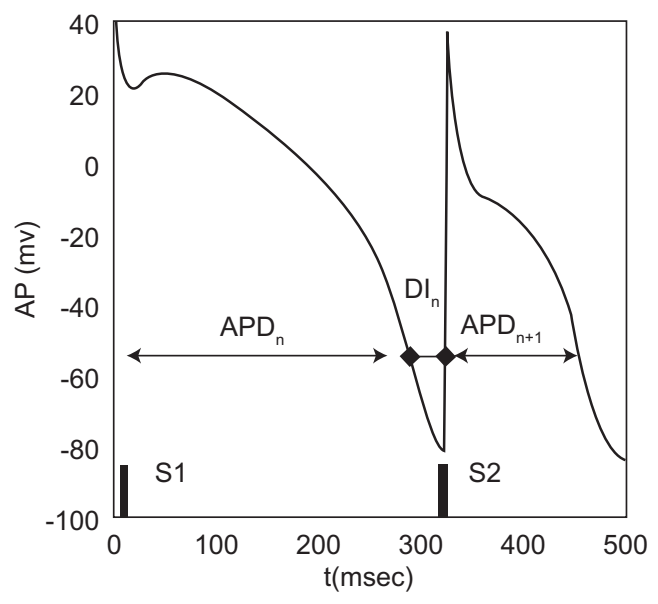

Figure 2: Illustration of the restitution property, APD and $\mathrm{DI}^{[8]}$

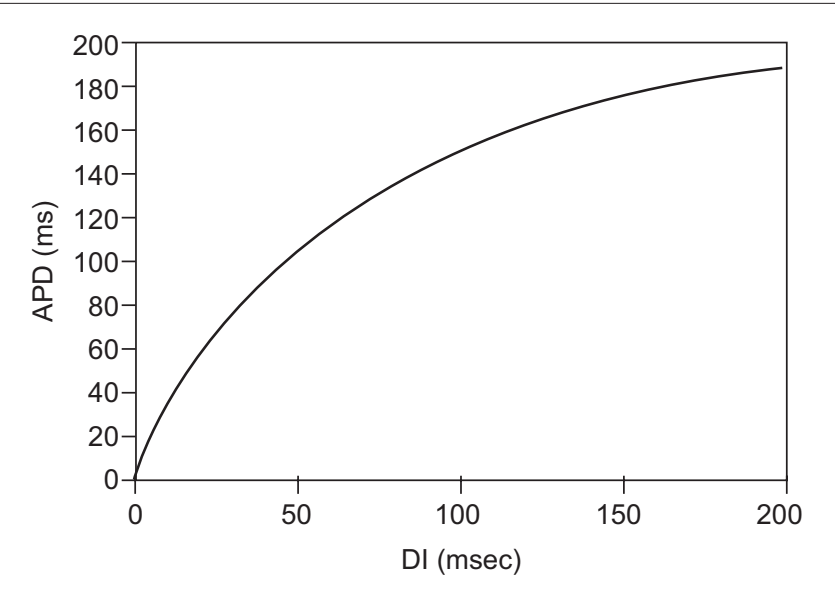

Figure 3: A sample restitution curve ${ }^{[10]}$

I. If $S^{\mathrm{t}}{ }_{\mathrm{mn}}$ is greater than the excitation threshold; $\Delta$ and $\mathrm{AP}_{\mathrm{mn}}^{\mathrm{t}}=\mathrm{R}_{\mathrm{mn}}^{\mathrm{t}}=0$, the cell in position $\mathrm{mn}$; cell ${ }_{\mathrm{mn}}$ will be excited in the next time step; $\mathrm{t}+1$ and $\mathrm{AP}_{\mathrm{mn}}^{\mathrm{t}+1}=\mathrm{AP}_{\text {up }}$ and $\mathrm{R}^{\mathrm{t}+1}=0$

II. If $\mathrm{S}_{\mathrm{mn}}^{\mathrm{t}}<\Delta$ and $\mathrm{R}_{\mathrm{mn}}^{\mathrm{t}}=\mathrm{AP}_{\mathrm{mn}}^{\mathrm{t}}=0$, the cell will not be stimulated and stays in its previous (rest) state

III. If $R_{m n}^{t}+A P_{m n}^{t} \neq 0$, the cell ${ }_{m n}$ will take the path in the state space, as shown in Figure 4. Notice that $R_{m n}$ starts decreasing when the $\mathrm{AP}_{\mathrm{mn}}$ reaches its maximum value After a complete evolution in the state space the $\mathrm{R}_{\mathrm{mn}}^{\mathrm{t}}$ and $\mathrm{AP}_{\mathrm{mn}}^{\mathrm{t}}$ will return to their rest states again 


\begin{tabular}{ll}
\hline Table I: Definition of parameters used in the proposed model \\
\hline Parameter name & Description \\
\hline$A P_{\text {up }}$ & AP up step \\
$A P_{\text {Down }}$ & AP down step \\
$A P_{d}$ & AP down step while R increases \\
$R_{\text {up }}$ & R up step \\
$R_{\text {Down }}$ & R down step \\
$\Delta$ & Cell's stimulus threshold \\
$N_{0}$ & The border between phase I and 2 in Figure 5 \\
$A P D_{\max }$ & The maximum value of APD \\
\hline
\end{tabular}

The parameter values in the model are set so that the time course of AP and R in a single cell are as depicted in Figure 5. Here the continued and dashed lines show AP and R, respectively. Referring to Figure 5, it is clear that the general morphology of action potential and refractory in a heart cell are preserved.

For the $\mathrm{R}$ state variable, five phases $(0,1,2,3,4)$ are assumed. In phase $0, R$ has its maximum value, so the cell is absolutely unexcitable. Phase 1 stands for $R$ when the cell has just entered the rest state. In this phase $R$ starts decreasing. Phases 0 and 1 are called absolute refractory. In phases 2 and 3 , R continues decreasing and a strong enough stimulus can re-excite the cell. In phase $4, \mathrm{R}$ reaches its minimum value; 0 . Here, the cell is in rest and normal stimulus can excite it and generate an AP with normal duration, that is, a normal APD.

If a cell is excited during phases 2 or 3, the restitution property will require that the APD is shorter than normal.

The main aim of this research was the simulation of restitution in a single cell, so a lattice of a single cell without any neighbor was considered. The automata rule was as discussed in section 3 and Figure 4 . The parameter $R_{\text {Down }}$ was considered as a time-dependent parameter, as:

$R_{\text {Down }}=\exp \left(\frac{-\alpha t}{N}\right)$

In eq. $4, t$ denotes time and $\alpha$ is an adjustable coefficient. The model was implemented by matlab.

\section{RESULTS}

The model parameters were adjusted so that by stimulating a single cell, the outcome of the AP and R morphology was generally similar to a ventricular cell. The outcome is depicted on the left side of Figure 6. Referring to Figure 6, it is clear that the proposed model can simulate the AP and $\mathrm{R}$ morphology.

In Figure 6, three stimuli are applied to a cell, at times 0 , 40 , and 70 milliseconds. The time interval between the first and second stimulus is long enough. In other words; $\mathrm{DI}_{1}$ is normal and the cell has enough time for full recovery, and

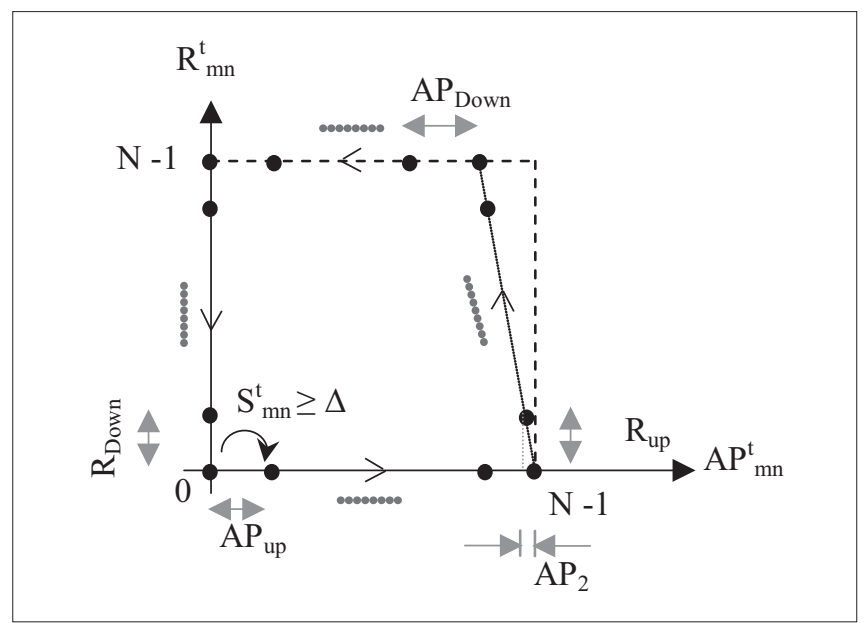

Figure 4: Evolution of the cell ${ }_{m n}$ state variables; $A P_{m n}$ and $R_{m n}$ in the state space. See the text for transition rules

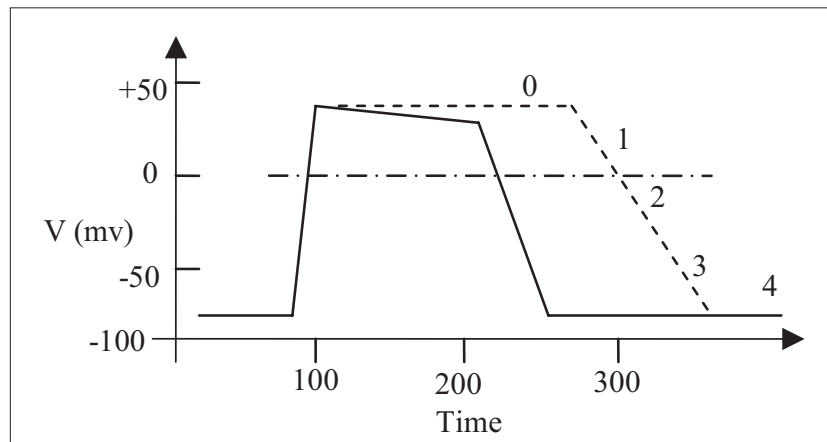

(a)

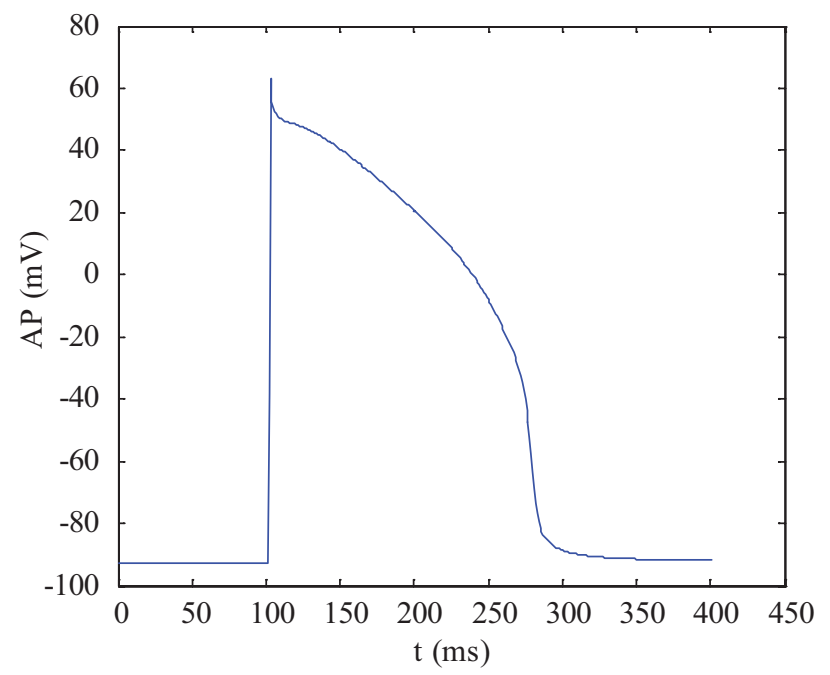

(b)

Figure 5: (a) Time course of AP and R as implemented in the model; (b) A real AP time course ${ }^{[1]}$

this leads to a normal $\mathrm{APD}_{2}$. The third stimulus is applied shortly after the second one. Therefore, $\mathrm{DI}_{2}$ is shorter than the normal case $\left(\mathrm{DI}_{1}\right)$. It means that the cell has not enough time for complete recovery. The right hand side of Figure 6 indicates that the proposed model can simulate the 


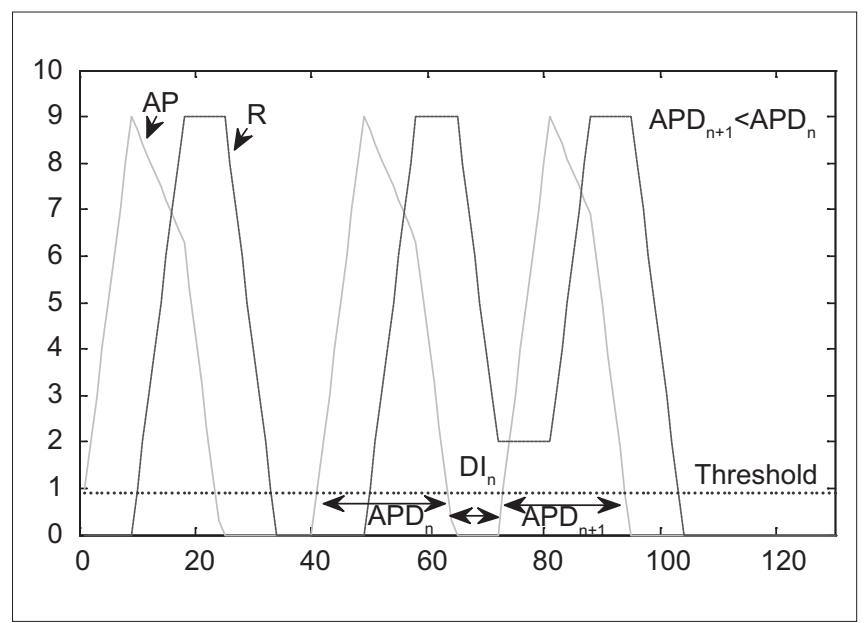

Figure 6: The outcome of the proposed model simulation. AP and R morphology are reserved as well as the restitution property

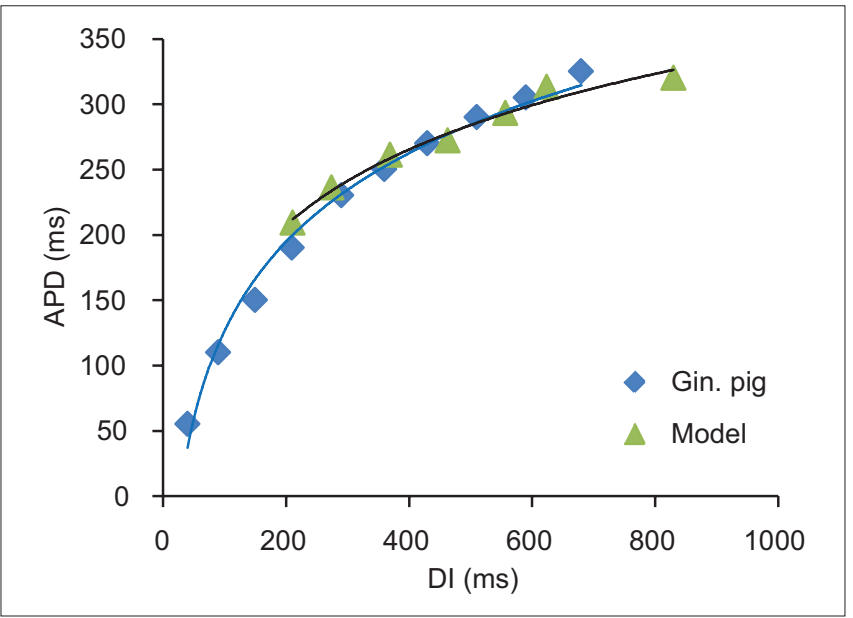

Figure 8: Comparison of the guinea pig restitution curve with the simulated curve created by the model. The model parameters are adjusted as in the table. The proposed model can simulate the guinea pig restitution curve

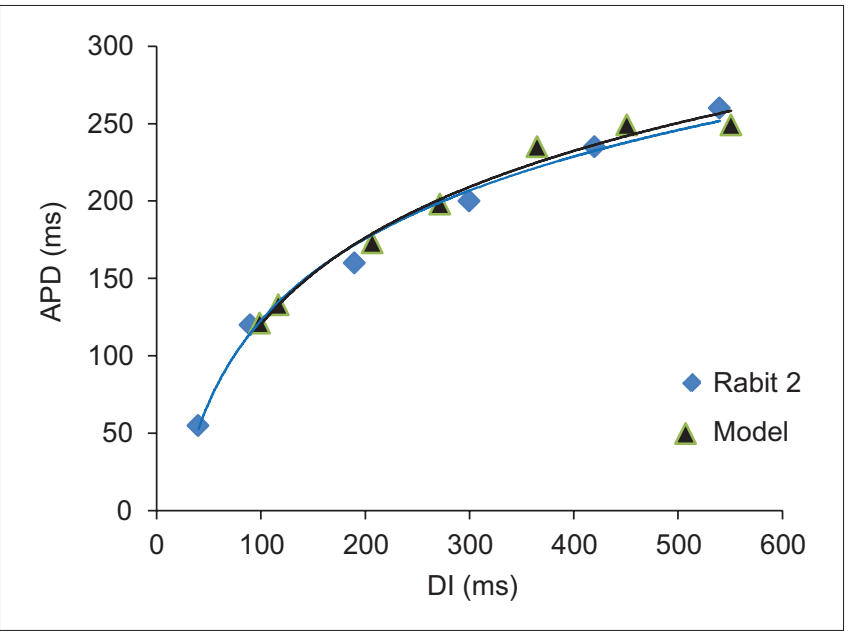

Figure I0: Comparison of the rabbit 2 restitution curve with the simulated curve created by the model. The model parameters are adjusted as shown in the table. It is clear that the proposed model can simulate the rabbit 2 restitution curve properly

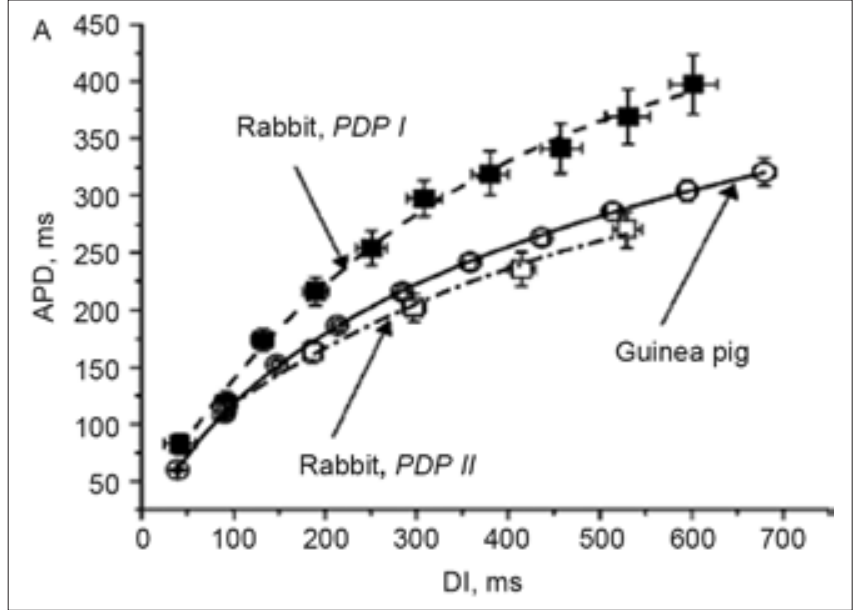

Figure 7: Three experimental restitution curves ${ }^{[12]}$

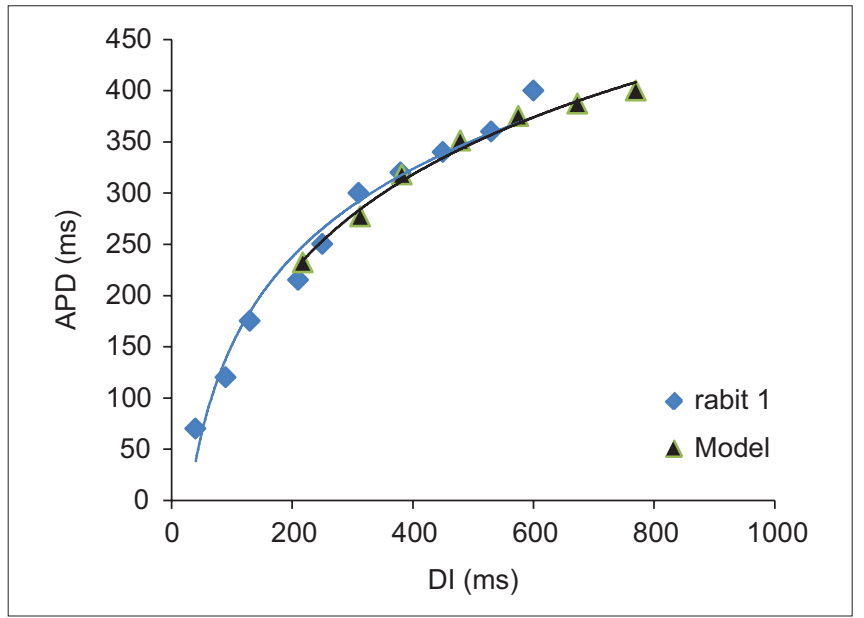

Figure 9: Comparison of the rabbit I restitution curve with the simulated curve created by the model. The model parameters are adjusted as in the table. It is clear that the proposed model can simulate the rabbit I restitution curve

restitution property, that is, the third APD $\left(\mathrm{APD}_{3}\right)$ is shorter than the first and second one.

After assurance about the installation of the restitution property in the model, its validity should be justified. Figure 7 depicts three actual restitution curves. ${ }^{[12]}$ If the proposed model is precise, it should simulate each of these curves by the adjustment of its parameters.

In this research the adjustment of the model was done via the regulation of $\mathrm{N}, \alpha$, and $\mathrm{APD}_{\max }$. The Normalized Mean Square Error (NMSE) criterion was used for fitness comparison of the restitution curves.

At first, the guinea pig restitution curve of Figure 7 was tested. The result is depicted in Figure 8. Here, the model parameters were adjusted as shown in the table in Figure 8. It is clear that the two curves coincided, with negligible NMSE. 
Second, as an attempt for the model validity justification, the rabbit 1 restitution curve of Figure 7 was examined. The result is depicted in Figure 9. Here, the model parameters were adjusted as shown in the Table in Figure 9. It is clear that the two curves coincided, with negligible NMSE.

And finally, the rabbit 2 restitution curve of Figure 7 was investigated. The outcome is illustrated in Figure 10. Here also, it is clear that the proposed model can simulate the restitution curve of rabbit 2 , when its parameters are adjusted as shown in the table of Figure 10.

\section{CONCLUSIONS}

Restitution is a particular property of the cardiac cell. In this research, a cellular automata-based model was proposed for simulating restitution. For the validation test of the model, the restitution curves that were simulated by the model were evaluated against the experimental results of the validated sources. In this research, the parameters $\alpha, \mathrm{N}$, and $\mathrm{APD}_{\max }$ were used for model regulation. The results showed that the proposed model could not only simulate restitution properly, but could also be regulated for simulating restitution curves of different kinds of cardiac cells. On account of the low computational load of the automata-based cell models, the model would be very useful for simulating propagation of electrical waves in the ventricular tissue and investigation of the wave break and fibrillation, which would form the matter of the authors' future researches.

\section{REFERENCES}

1. K. H. ten Tusscher, and A. V. Panfilov. Cell model for efficient simulation of wave propagation in human ventricular tissue under normal and pathological conditions. Phys Med Biol 51, pp. 6141-56, 2006.

2. A. Garfinkel, Y. H. Kim, O. Voroshilovsky, Z. Qu, J. R. Kil, M. H. Lee, et al. Preventing ventricular fibrillation by flattening cardiac restitution. Proc Natl Acad Sci U.S.A. 97, pp. 6061-6, 2000.

3. M. L. Koller, M. L. Riccio, and R. F. Gilmour. Dynamic restitution of action potential duration during electrical alternans and ventricular fibrillation. Am J Physiol 275, pp. 1635-42, 1998.

4. W. H. Steeb. The nolinear workbook. $3^{\text {rd }}$ ed. Singapore: World Scientific Publishing Company; pp. 244-56, 2005.

5. W. E. Yu, and R. P. Saldãna. Computational aspects of modeling excitable media using cellular automata, Proceedings of the Second National Workshop and Conference on Modeling, Simulation, and Scientific Computing, 2001.

6. C. L. Chang, Y. J. Zhang, and Y. Y. Gdong. Cellular automata for edge detection of images. Proceedings of the Third International Conference on Machine Learning and Cybernetics, Shanghai, Aug 26-29, 2004.

7. Y. Zhao, and S. A. Billings. Neighborhood detection using mutual information for the identification of cellular automata. IEEE Trans Syst Man Cybern B Cybern 36, pp. 473-9, 2006.

8. D. Mocanu, M. Morega, and A. Morega. A Simulation study of electrical rhythms in a paced cardiac fiber. Proceedings of the Fifth Workshop on Mathematical Modelling of Environmental and Life Sciences Problems Constant, Romania. pp.153-62, Sept 2006.

9. D. G. Schaeffer, W. Ying, and X. Zhao. Asymptotic approximation of an ionic model for cardiac restitution. Nonlinear Dyn 51, pp. 189-98, 2008.

10. F. A. Atienza, J. R. Carrion, A. G. Alberola, J. L. Alvarez, J. J.Munoz, J. M. Sanchez, et al. A probabilistic model of cardiac electrical activity based on a cellular automata system. Rev Esp Cardiol 58, pp. 41-7, 2005.

11. Available from: http://cor.physiol.ox.ac.uk/ (last accessed @ Feb. 2012)

12. E. G. Tolkacheva, J. M. Anumonwo, and J. Jalife. Action potential duration restitution portraits of mammalian ventricular myocytes: Role of calcium current. Biophys J 91, pp. 2735-45, 2006.

How to cite this article: Sabzpoushan SH, Pourhasanzade F. A cellular automata-based model for simulating restitution property in a single heart cell. J Med Sign Sens 2011;1:19-23

Source of Support: Nil, Conflict of Interest: None declared

\section{BIOGRAPHIES}

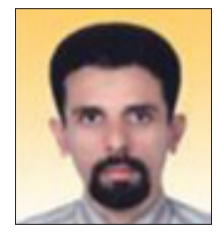

Seyed Hojjat Sabzpoushan is assistant professor in Biomedical Engineering. He is with the Department of Biomedical Engineering and the research laboratory of biomedical signals and sensors, Iran University of Science and Technology (I.U.S.T.), Tehran, Iran.

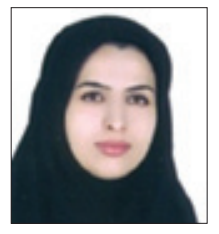

Fateme Pourhasanzade is a phD student in biomedical engineering department; Iran University of Science and Technology (I.U.S.T.), Tehran, Iran. 